\title{
RLS Wiener FIR Predictor and Filter Based on Innovation Approach in Linear Discrete-Time Stochastic Systems
}

\author{
Seiichi Nakamori \\ Specially Appointed Professor, Department of Technology, Faculty of Education, Kagoshima University, \\ Kourimoto, Kagoshima, 890-0065 Japan \\ Email: k6165161@kadai.jp
}

\begin{abstract}
This paper designs the recursive least-squares (RLS) Wiener finite impulse response (FIR) predictor and filter, based on the innovation approach, in linear discrete-time stochastic systems. It is assumed that the signal is observed with additive white noise and the signal process is uncorrelated with the observation noise process. This paper also presents the recursive algorithms for the estimation error variance functions of the proposed RLS Wiener FIR predictor and filter. A numerical simulation example shows the estimation characteristics of the RLS Wiener FIR predictor and filter. Specifically, the estimation characteristics of the proposed RLS Wiener FIR filter and predictor are compared with those of the existing RLS Wiener FIR filter and the RLS Wiener predictor, derived based on the existing RLS Wiener filter, respectively.
\end{abstract}

Keywords: FIR predictor, FIR filter, discrete-time stochastic systems, innovation approach, WienerHopf equation.

\section{Introduction}

In [1], the finite impulse response (FIR) filter and smoother are proposed, given continuous time-invariant state-space models. The FIR estimation algorithms calculate the Riccati-type differential equations on a finite interval. In comparison with the conventional recursive filter which necessitates observed value at each time increasingly, the FIR filter has the property of improving filter divergence due to modeling errors and the signals in systems under sudden changes [2], [3]. Jazwinski [2] and Schweppe [4] devise the FIR filter, given discrete-time state-space models without input noise. Bruckstein and Kailath [5] present the recursive FIR filter, provided that the state-space models with input noise in both continuous-time and discrete-time stochastic systems. In [6]-[8], receding horizon Kalman FIR filtering algorithms are proposed in continuous-time and discrete-time stochastic systems. Also, the $H_{2}$ FIR smoother [9] and the $H_{\infty}$ FIR smoother [10] are presented, given discrete-time state-space models. Alternatively to the Kalman estimator, which necessitates the state-space model, the filter, the fixed-point smoother [11] and the fixed-lag smoother [12] are proposed, under the condition that the covariance information of the signal and observation noise processes is given, in linear continuous-time stochastic systems. In [11] and [12], the auto-covariance function of the signal process is expressed in the form of the semi-degenerate kernel. In [13], the recursive least-squares (RLS) Wiener FIR filtering algorithm is presented in linear discrete-time stochastic systems. In [14], by using the covariance information, the RLS-FIR filter is designed in linear continuous-time stochastic systems. In [14], the auto-covariance function of the signal process is expressed in the form of the semi-degenerate kernel. Also, in linear discrete-time stochastic systems, the RLS Wiener FIR fixed-lag smoothing and filtering algorithms [15] are proposed. In [15], the typos in the RLS Wiener FIR filtering algorithm [13] are corrected. In [16], the RLS-FIR smoother is presented in estimating the signal at each start time of the finite interval in linear continuous-time stochastic systems. Here, the auto-covariance function of the signal process is expressed in the semi-degenerate kernel form.

In comparison with the Kalman estimators, the RLS Wiener estimators do not necessitate the information of the input noise variance and the input matrix in the state-space model. The RLS Wiener estimators do not bring unnecessary estimation errors caused by the approximations of the input noise variance and the input matrix. In the estimation problems, the filtering, smoothing and prediction algorithms are interesting [17]. Compared with the RLS Wiener estimators, by using updated observed values, the RLS Wiener FIR estimators calculate the estimates of the signal recursively in terms of 
observed values in the finite interval. This paper, in particular, focuses on the new designs of the RLS Wiener FIR predictor and filter, based on the innovation approach, in linear discrete-time stochastic systems. Specifically, this paper newly derives the RLS Wiener FIR filtering and prediction algorithms which have feasible estimation characteristics and reduce the computation times in comparison with the existing RLS Wiener FIR filter [15] and the RLS Wiener FIR predictor, obtained based on the RLS Wiener filter in [15], respectively. It is assumed that the signal process is uncorrelated with the observation noise process. In accordance with the stability of the proposed RLS Wiener FIR predictor and filter, this paper also presents the recursive algorithms for the estimation error variance functions of the RLS Wiener FIR predictor and filter.

Section 3 presents the RLS Wiener FIR prediction and filtering algorithms in Theorem 1. Section 4 discusses on the stability of the proposed RLS Wiener FIR predictor and filter based on the prediction and filtering error variance functions. Section 5 shows a numerical simulation example of the proposed RLS Wiener FIR predictor and filter. The estimation characteristics of the proposed RLS Wiener FIR filter and predictor are compared with those of the existing RLS Wiener FIR filter [15] and the RLS Wiener predictor, derived based on the RLS Wiener filter in [15].

Section 2 introduces the preliminary formulation on the FIR prediction and filtering problems based on the innovation approach.

\section{RLS Wiener Prediction and Filtering Problems}

Let the time-invariant state equation and the observation equation be represented by

$$
\begin{aligned}
& x(k+1)=\Phi x(k)+w(k), \\
& y(k)=z(k)+v(k), z(k)=H x(k),
\end{aligned}
$$

in linear discrete-time stochastic systems. Here, $k \in R$ denotes time, $x(k) \in R_{N}$ is the state vector, $w(k) \in R_{N}$ is white input noise, $y(k) \in R_{M}$ is the observed value, $\Phi \in R_{N \times N}$ is the state-transition matrix, $H \in R_{M \times N}$ is the observation matrix, $z(k) \in R_{M}$ is the signal vector and $v(k) \in R_{M}$ is white observation noise. Let the auto-covariance functions of $w(k)$ and $v(k)$ be given by

$$
\begin{aligned}
& E\left[w(k) w^{T}(s)\right]=Q \delta_{K}(k-s), Q>0, \\
& E\left[v(k) v^{T}(s)\right]=R \delta_{K}(k-s), R>0 .
\end{aligned}
$$

Here, $\delta_{K}(\cdot)$ denotes the Kronecker $\delta$ function. It is assumed that the signal and observation noise are mutually independent stochastic processes with zero means.

Let $K(k, s)$ represent the auto-covariance function of the state vector and let $K(k, s)$ be expressed in the semi-degenerate kernel form [15] of

$$
\begin{aligned}
& K(k, s)=\left\{\begin{array}{l}
\alpha(k) \beta^{T}(s), 0 \leq s \leq k, \\
\beta(k) \alpha^{T}(s), 0 \leq k \leq s,
\end{array}\right. \\
& \alpha(k)=\Phi^{k}, \beta^{T}(k)=\Phi^{-k} K(k, k) .
\end{aligned}
$$

Here, $\alpha(k)$ and $\beta(s)$ are bounded square matrices of order $N$.

Let a $m$-step ahead FIR prediction estimate $\hat{x}(k+m \mid k-L+1, k)$ of the state vector $x(k+m)$ be given by

$$
\begin{aligned}
& \hat{x}(k+m \mid k-L+1, k)=\sum_{i=k-L+1}^{k} g(k, i) \nu(i), \\
& \nu(i)=y(i)-H \Phi \hat{x}_{f}(i-1 \mid i-1-L+1, i-1),
\end{aligned}
$$

as a linear transformation of the innovation process $\nu(i), k-L+1 \leq i \leq k$, of finite interval $L$. Here, $g(k, i)$ is referred to as the impulse response function and let $\hat{x}_{f}(i-1 \mid i-1-L+1, i-1)$ represent the FIR filtering estimate of $x(i-1)$. By introducing an impulse response function $g_{f}(k, i)$, the FIR filtering estimate $\hat{x}_{f}(k \mid k-L+1, k)$ of $x(k)$ is expressed as

$$
\hat{x}_{f}(k \mid k-L+1, k)=\sum_{i=k-L+1}^{k} g_{f}(k, i) \nu(i)
$$


based on the innovation process $\nu(i), k-L+1 \leq i \leq k$.

The impulse response function, which minimizes the mean-square value of the FIR prediction error $x(k+m)-\hat{x}(k+m \mid k-L+1, k)$,

$$
J=E\left[\|x(k+m)-\hat{x}(k+m \mid k-L+1, k)\|^{2}\right],
$$

satisfies

$$
E\left[x(k+m) \nu^{T}(s)\right]=\sum_{i=k-L+1}^{k} g(k, i) E\left[\nu(i) \nu^{T}(s)\right] d \tau
$$

by an orthogonal projection lemma [17]

$$
x(k+m)-\hat{x}(k+m \mid k-L+1, k) \perp \nu(s), k-L+1 \leq s \leq k .
$$

Here, " $\perp$ " denotes the notation of the orthogonality. From (1), (2) and (8), the impulse response function $g(k, s)$, for the linear RLS Wiener FIR prediction estimate, satisfies

$$
g(k, s) \Lambda(s)=K(k+m, s) H^{T}-\sum_{i=s-1-L+1}^{s-1} g(k, i) \Lambda(i) g_{f}^{T}(s-1, i) \Phi^{T} H^{T} .
$$

Here, $\Lambda(s)$ is the auto-variance function of the innovation process and is given by $\Lambda(s)=E\left[\nu(s) \nu^{T}(s)\right]$. Similarly to the derivation of (9), the impulse response function $g_{f}(k, s)$, which yields the RLS Wiener FIR filtering estimate in (5), satisfies

$$
g_{f}(k, s) \Lambda(s)=K(k, s) H^{T}-\sum_{i=s-1-L+1}^{s-1} g_{f}(k, i) \Lambda(i) g_{f}^{T}(s-1, i) \Phi^{T} H^{T} .
$$

Based on the preliminary formulation on the RLS Wiener FIR prediction and filtering problems, the RLS Wiener FIR prediction and filtering algorithms are proposed in section 3.

\section{RLS Wiener FIR Prediction and Filtering Algorithms}

Starting with (9) and (10) for the optimal impulse response functions, we derive the RLS Wiener FIR prediction and filtering algorithms for the signal based on the invariant imbedding method [15], [16]. Theorem 1 presents the RLS Wiener FIR prediction and filtering algorithms.

Theorem 1 Let the state equation and the observation equation be given by (1) in linear time-invariant stochastic systems. Let the auto-covariance function of the state vector $x(k)$ be given by (3) in the semi-degenerate kernel form. Then the algorithms for the RLS Wiener FIR prediction and filtering estimates consist of (11)-(17).

m-step ahead RLS Wiener FIR prediction estimate of the signal $z(k+m): \hat{z}(k+m \mid k-L+1, k)$

$$
\hat{z}(k+m \mid k-L+1, k)=H \hat{x}(k+m \mid k-L+1, k)
$$

m-step ahead RLS Wiener FIR prediction estimate of the state vector $x(k+m): \hat{x}(k+m \mid k-L+1, k)$

$$
\hat{x}(k+m \mid k-L+1, k)=\Phi^{m} \hat{x}_{f}(k \mid k-L+1, k)
$$

RLS Wiener FIR filtering estimate of the signal $z(k): \hat{z}(k \mid k-L+1, k)$

$$
\hat{z}(k \mid k-L+1, k)=H \hat{x}_{f}(k \mid k-L+1, k)
$$

RLS Wiener FIR filtering estimate of the state vector $x(k): \hat{x}_{f}(k \mid k-L+1, k)$

$$
\begin{aligned}
& \hat{x}_{f}(k \mid k-L+1, k)=\Phi \hat{x}_{f}(k-1 \mid k-1-L+1, k-1) \\
& +g_{f}(k, k)\left(y(k)-H \Phi \hat{x}_{f}(k-1 \mid k-1-L+1, k-1)\right) \\
& -\Phi^{L} g_{f}(k-L, k-L)\left(y(k-L)-H \Phi \hat{x}_{f}(k-L-1 \mid k-L-1-L+1, k-L-1)\right)
\end{aligned}
$$


(Initial condition of $\hat{x}_{f}(k \mid k-L+1, k)$ at $k=L: \hat{\bar{x}}_{f}(L \mid 1, L)$ )

Filter gain: $g_{f}(k, k)$

$$
g_{f}(k, k)=\left(K(k, k) H^{T}-\Phi S(k-1) \Phi^{T} H^{T}\right) \Lambda^{-1}(k)
$$

Variance function of the innovation process $\nu(k): \Lambda(k)$

$$
\Lambda(k)=\left(H K(k, k) H^{T}+R-H \Phi S(k-1) \Phi^{T} H^{T}\right)
$$

Variance function of the filtering estimate $\hat{x}_{f}(k, k): S(k)$

$$
\begin{aligned}
& S(k)=\Phi S(k-1) \Phi^{T}+g_{f}(k, k) \Lambda(k) g_{f}^{T}(k, k) \\
& -\Phi^{L} g_{f}(k-L, k-L) \Lambda(k-L) g_{f}^{T}(k-L, k-L)\left(\Phi^{T}\right)^{L}
\end{aligned}
$$

(Initial condition of $S(k)$ at $k=L: \bar{S}(L)$ )

Initial conditions $\hat{\bar{x}}_{f}(L \mid 1, L)$ and $\bar{S}(L)$, required in the difference equations (14) and (17) respectively for the RLS Wiener FIR filtering estimate $\hat{x}_{f}(k \mid k-L+1, k)$ and the variance function of the filtering estimate $\hat{x}_{f}(k, k), S(k)$, are calculated by (18)-(20) recursively.

Filtering estimate of $x(L): \hat{\bar{x}}_{f}(L \mid 1, L)$

$$
\begin{aligned}
& \hat{\bar{x}}_{f}(L \mid 1, L)=\Phi \hat{\bar{x}}_{f}(L-1 \mid 1, L-1)+\bar{g}_{f}(L, L)\left(y(L)-H \Phi \hat{\bar{x}}_{f}(L-1 \mid 1, L-1)\right), \\
& \hat{\bar{x}}_{f}(0 \mid 1,0)=0
\end{aligned}
$$

Filter gain: $\bar{g}_{f}(L, L)$

$$
\begin{aligned}
& \bar{g}_{f}(L, L)=\left(K(L, L) H^{T}-\Phi \bar{S}(L-1) \Phi^{T} H^{T}\right) \bar{\Lambda}^{-1}(L), \\
& \bar{\Lambda}(L)=H K(L, L) H^{T}+R-H \Phi \bar{S}(L-1) \Phi^{T} H^{T}
\end{aligned}
$$

Variance function of the filtering estimate $\hat{\bar{x}}_{f}(L \mid 1, L): \bar{S}(L)$

$$
\begin{aligned}
& \bar{S}(L)=\Phi \bar{S}(L-1) \Phi^{T}+\bar{g}_{f}(L, L) \bar{\Lambda}(L) \bar{g}_{f}^{T}(L, L), \\
& \bar{S}(0)=0
\end{aligned}
$$

Proof of Theorem 1 is deferred to the Appendix.

It is seen, based on the RLS Wiener FIR filter [15], that the $m$-step ahead RLS Wiener prediction estimates of the signal $z(k)$ and the state vector $x(k)$ are calculated by (11) and (12). Here, the filtering estimate of the state vector $x(k)$ by the RLS Wiener FIR filter in [15] is substituted into $\hat{x}_{f}(k \mid k-L+1, k)$ in (12).

The number of the difference equations included in the current RLS Wiener FIR filter is $N(N+1) / 2+N$ in comparison with $5 N^{2}+2 N$ in the RLS Wiener FIR filter [15]. As for the calculations of the initial conditions, the proposed RLS Wiener FIR filtering algorithm includes $N(N+1) / 2+N$ difference equations against $6 N^{2}+2 N$ in [15]. Hence, in the calculations of the estimates, the proposed RLS Wiener FIR filter and predictor need less computation times than the RLS Wiener FIR filter in [15] and the RLS Wiener FIR predictor, obtained based on the RLS Wiener FIR filter in [15], respectively.

Section 4 presents the algorithms for the estimation error variance functions of the proposed RLS Wiener FIR predictor and filter.

\section{Estimation Error Variance Functions of RLS Wiener FIR Predictor and Filter}

By referring to Theorem 1, the estimation error variance function $P_{\tilde{z}}(k+m)$ of the proposed RLS Wiener FIR predictor is formulated as follows.

$$
\begin{aligned}
& P_{\tilde{z}}(k+m)=E\left[(z(k+m)-\hat{z}(k+m \mid k-L+1, k))(z(k+m)-\hat{z}(k+m \mid k-L+1, k))^{T}\right] \\
& =H K(k+m, k+m) H^{T}-H E\left[\hat{x}(k+m \mid k-L+1, k) \hat{x}^{T}(k+m \mid k-L+1, k)\right] H^{T} \\
& =H K(k+m, k+m) H^{T}-H \Phi^{m} E\left[\hat{x}(k \mid k-L+1, k) \hat{x}^{T}(k \mid k-L+1, k)\right]\left(\Phi^{T}\right)^{m} H^{T} \\
& =H K(k+m, k+m) H^{T}-H \Phi^{m} S(k)\left(\Phi^{T}\right)^{m} H^{T}
\end{aligned}
$$


Here, the auto-variance function $S(k)$ of the filtering estimate $\hat{x}(k \mid k-L+1, k)$ is calculated by (17) recursively. The auto-variance function $P_{\hat{z}}(k+m)$ of the RLS Wiener FIR prediction estimate $\hat{z}(k+$ $m \mid k-L+1, k)$ is given by $H \Phi^{m} S(k)\left(\Phi^{T}\right)^{m} H^{T}$. Since the RLS Wiener FIR prediction error variance function $P_{\tilde{z}}(k+m)$ is the positive semi-definite matrix, it is seen that $P_{\hat{z}}(k+m)$ is upper bounded by $H K(k+m, k+m) H^{T}$ and lower bounded by the zero matrix as

$$
0 \leq P_{\hat{z}}(k+m) \leq H K(k+m, k+m) H^{T}, P_{\hat{z}}(k+m)=H \Phi^{m} S(k)\left(\Phi^{T}\right)^{m} H^{T} .
$$

(21) indicates that the proposed RLS Wiener FIR predictor is stable, provided that the function $H K(k+$ $m, k+m) H^{T}$ is bounded. Similarly to the RLS Wiener FIR prediction error variance function $P_{\tilde{z}}(k+m)$, from the RLS Wiener FIR filtering error variance function $P_{\tilde{z}}(k)$, given by $H K(k, k) H^{T}-H S(k) H^{T}$, the auto-variance function $P_{\hat{z}}(k)$ of the RLS Wiener FIR filtering estimate $\hat{z}(k \mid k-L+1, k)$ is upper bounded by $H K(k, k) H^{T}$ and lower bounded by the zero matrix as

$$
0 \leq P_{\hat{z}}(k) \leq H K(k, k) H^{T}, P_{\hat{z}}(k)=H S(k) H^{T} .
$$

(22) indicates that the proposed RLS Wiener FIR filter is stable, under the condition that the function $H K(k, k) H^{T}$ is bounded.

Section 5 shows a numerical simulation example of the proposed RLS Wiener FIR prediction and filtering algorithms.

\section{A Numerical Simulation Example}

Let a scalar observation equation be given by

$$
y(k)=z(k)+v(k), z(k)=H x(k) .
$$

Here, $H$ represents the observation vector and $x(k)$ represents the state vector. Let the observation noise $v(k)$ be a zero-mean white Gaussian process with the variance $R, N(0, R)$. Let the auto-covariance function of the signal $z(k)$ be given by

$$
K_{z}(k, s)=H \Phi^{k-s} K(s, s) H^{T}, 0 \leq s \leq k,
$$

where $K(s, s)=K(0)$ represents the variance of the state vector $x(s)$ in wide-sense stationary stochastic systems and $\Phi$ represents the system matrix.

Let us consider the signal $z(k)$, which is generated by the second-order autoregressive (AR) model.

$$
\begin{aligned}
& z(k+1)=-a_{1} z(k)-a_{2} z(k-1)+w(k), E[w(k) w(s)]=\sigma^{2} \delta_{K}(k-s), \\
& a_{1}=-0.1, \quad a_{2}=-0.8, \quad \sigma=0.5 .
\end{aligned}
$$

Here, the state-space model for $z(k)$ is given by

$$
\begin{aligned}
& z(k)=H x(k)=x_{1}(k), H=[10], x(k)=\left[\begin{array}{l}
x_{1}(k) \\
x_{2}(k)
\end{array}\right], \\
& {\left[\begin{array}{l}
x_{1}(k+1) \\
x_{2}(k+1)
\end{array}\right]=\left[\begin{array}{cc}
0 & 1 \\
-a_{2}-a_{1}
\end{array}\right]\left[\begin{array}{l}
x_{1}(k) \\
x_{2}(k)
\end{array}\right]+\left[\begin{array}{l}
0 \\
1
\end{array}\right] w(k) .}
\end{aligned}
$$

In this case, the auto-covariance function $K_{z}(\cdot)$ of the signal $z(k)$ is given by [18]

$$
\begin{aligned}
& K_{z}(0)=\sigma^{2}, \\
& K_{z}(m)=\sigma^{2} \frac{\alpha_{1}\left(\alpha_{2}^{2}-1\right) \alpha_{1}^{m}}{\left(\alpha_{2}-\alpha_{1}\right)\left(\alpha_{2} \alpha_{1}+1\right)}-\frac{\alpha_{2}\left(\alpha_{1}^{2}-1\right) \alpha_{2}^{m}}{\left(\alpha_{2}-\alpha_{1}\right)\left(\alpha_{2} \alpha_{1}+1\right)}, 0<m, \\
& \alpha_{1}, \alpha_{2}=\frac{-a_{1} \pm \sqrt{a_{1}^{2}-4 a_{2}}}{2} .
\end{aligned}
$$

Hence, with regards to the calculations of the RLS Wiener FIR prediction and filtering algorithms, the necessary quantities are as follows.

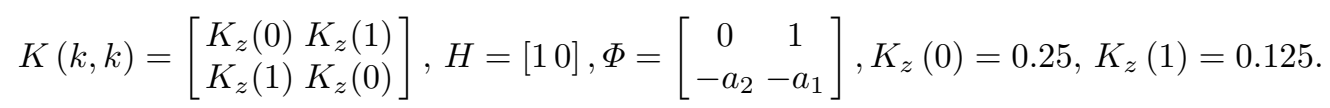


If we substitute (28) into the RLS Wiener FIR prediction and filtering algorithms of Theorem 1, we can calculate the prediction and filtering estimates recursively. Fig.1 illustrates the signal $z(k)$ and the RLS Wiener FIR one-step ahead prediction estimate $\hat{z}(k+1 \mid k-L+1, k)$ vs. $k, 30<k<200$, for the finite interval $L=30$ and the white Gaussian observation noise $N\left(0,0.1^{2}\right)$. Fig.2 illustrates the mean-square values (MSVs) of the one-step ahead prediction errors $z(k+1)-\hat{z}(k+1 \mid k-L+1, k)$ by the RLS Wiener FIR predictor vs. the finite interval $\mathrm{L}$ for the white Gaussian observation noises $N\left(0,0.1^{2}\right), N\left(0,0.3^{2}\right)$, $N\left(0,0.5^{2}\right)$ and $N\left(0,0.7^{2}\right)$. For the finite interval $L$, less than or equal to $L=90$, as $\mathrm{L}$ becomes large, the MSVs by the RLS Wiener FIR predictor decrease. At around $L=90$, the MSV has the smallest value for the respective observation noise. Here, the MSV of the RLS Wiener FIR prediction errors is calculated by $\sum_{k=L}^{L+299}\left(z(k+1)-\hat{z}(k+1 \mid k-L+1, k)^{2} / 300\right.$. Fig.3 illustrates the MSVs of the prediction errors $z(k+m)-\hat{z}(k+m \mid k-L+1, k), L=50$, vs. the prediction step $m, 1 \leq m \leq 5$, by the proposed RLS Wiener FIR predictor and the MSVs of the filtering errors $z(k)-\hat{z}(k \mid k-\bar{L}+1, k)$, in the case of $m=0$, by the proposed RLS Wiener FIR filter for the white Gaussian observation noises $N\left(0,0.1^{2}\right), N\left(0,0.3^{2}\right)$, $N\left(0,0.5^{2}\right)$ and $N\left(0,0.7^{2}\right)$. In Fig. 3 , as the prediction step increases, the prediction accuracy is degraded for each observation noise. Fig.4 illustrates the MSVs of the prediction errors $z(k+m)-\hat{z}(k+m \mid k-L+1, k)$, $L=100$, vs. the prediction step $m, 1 \leq m \leq 5$, by the proposed RLS Wiener FIR predictor and the MSVs of the filtering errors $z(k)-\hat{z}(k \mid k-L+1, k)$, in the case of $m=0$, by the proposed RLS Wiener FIR filter for the white Gaussian observation noises $N\left(0,0.1^{2}\right), N\left(0,0.3^{2}\right), N\left(0,0.5^{2}\right)$ and $N\left(0,0.7^{2}\right)$. In Fig.4, as the prediction step increases, the prediction accuracy is degraded for each observation noise. From Fig.3 and Fig.4, it is seen that the prediction accuracy for the finite interval $L=100$ is superior to the case of $L=50$. Fig. 5 illustrates the MSVs of the filtering errors $z(k)-\hat{z}(k \mid k-L+1, k)$ vs. the finite interval $L$ by the proposed RLS Wiener FIR filter for the white Gaussian observation noises $N\left(0,0.1^{2}\right)$, $N\left(0,0.3^{2}\right), N\left(0,0.5^{2}\right)$ and $N\left(0,0.7^{2}\right)$. Here, the MSV of the RLS Wiener filtering errors is calculated by $\sum_{k=L}^{L+299}\left(z(k)-\hat{z}(k \mid k-L+1, k)^{2} / 300\right.$. In Fig.5, as the finite interval L increases, the filtering accuracy is improved for each observation noise. At around $L=90$, the MSV has the smallest value for the respective observation noise. Fig.6 illustrates the MSVs of the filtering errors $z(k)-\hat{z}(k \mid k-L+1, k)$ vs. the finite interval $L$ by the RLS Wiener FIR filter in [15] for the white Gaussian observation noises $N\left(0,0.1^{2}\right)$, $N\left(0,0.3^{2}\right), N\left(0,0.5^{2}\right)$ and $N\left(0,0.7^{2}\right)$. In Fig.6, the MSV for the observation noise $N\left(0,0.3^{2}\right)$ shows abrupt increase at the finite interval $L=30$. In Fig.5 and Fig.6, the MSVs of the filtering errors at around $L=90$ are almost same. From Fig.5, the proposed RLS Wiener FIR filter shows smooth decrease of the MSV of the filtering errors as the finite interval $L$ increases in comparison with Fig.6. This shows that the proposed RLS Wiener FIR filter has the feasible estimation property. Fig.7 shows the MSVs of the one-step ahead prediction errors $z(k+1)-\hat{z}(k+1 \mid k-L+1, k)$ by the RLS Wiener FIR predictor, obtained based on the RLS Wiener FIR filter in [15], vs. the finite interval $L$ for the white Gaussian observation noises $N\left(0,0.1^{2}\right), N\left(0,0.3^{2}\right), N\left(0,0.5^{2}\right)$ and $N\left(0,0.7^{2}\right)$. In Fig. 7 , the MSV for the observation noise $N\left(0,0.3^{2}\right)$ shows abrupt increase at the finite interval $L=30$. In Fig.2 and Fig.7, the MSVs of the prediction errors at around $L=90$ are almost same. From Fig.2, the proposed RLS Wiener FIR predictor shows smooth decrease of the MSV of the prediction errors as the finite interval $L$ increases in comparison with Fig.7. This shows that the proposed RLS Wiener FIR predictor has the feasible estimation property.

\section{Conclusions}

This paper has proposed the RLS Wiener FIR predictor and filter, based on the innovation approach, in linear discrete-time stochastic systems. This paper also has presented the recursive algorithms for the estimation error variance functions of the proposed RLS Wiener FIR predictor and filter. A numerical simulation example has shown the estimation characteristics of the proposed RLS Wiener FIR predictor and filter. From Fig. 2 and Fig.5, at around the finite interval $L=90$, the MSVs of the one-step ahead prediction and filtering errors have the smallest values for the respective observation noise. Specifically, from Fig.5 and Fig.6, the proposed RLS Wiener FIR filter has the feasible estimation property. Also, from Fig.2 and Fig.7, the proposed RLS Wiener FIR predictor shows the feasible estimation property.

Furthermore, the number of the difference equations included in the current RLS Wiener FIR filter is $N(N+1) / 2+N$ in comparison with $5 N^{2}+2 N$ in the RLS Wiener FIR filter [15]. As for the calculations of 


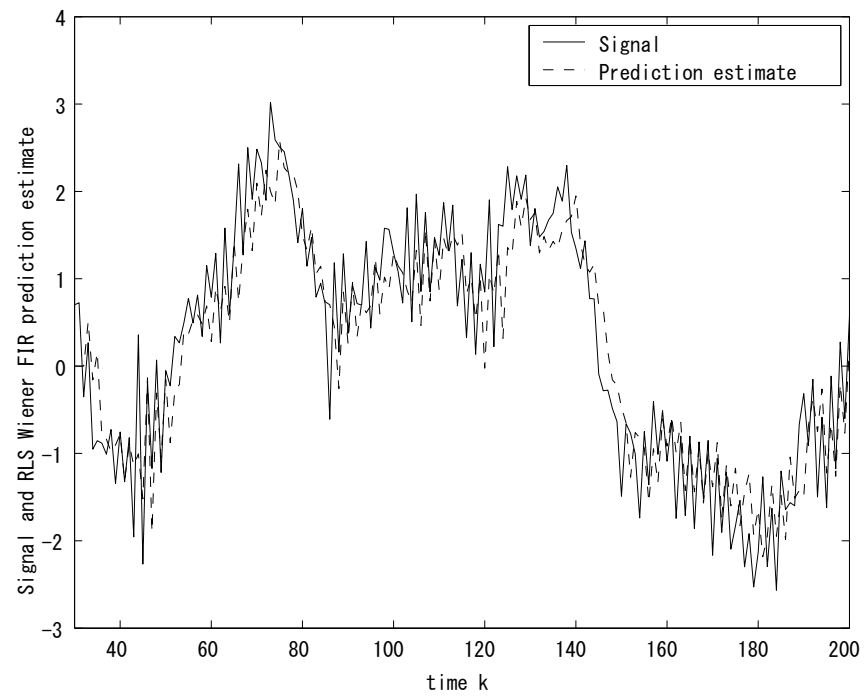

Figure 1. Signal $z(k)$ and the RLS Wiener FIR one-step ahead prediction estimate $\hat{z}(k+1 \mid k-L+1, k)$ vs. $k$, $30<k<200$, for the finite interval $L=30$ and the white Gaussian observation noise $N\left(0,0.1^{2}\right)$.

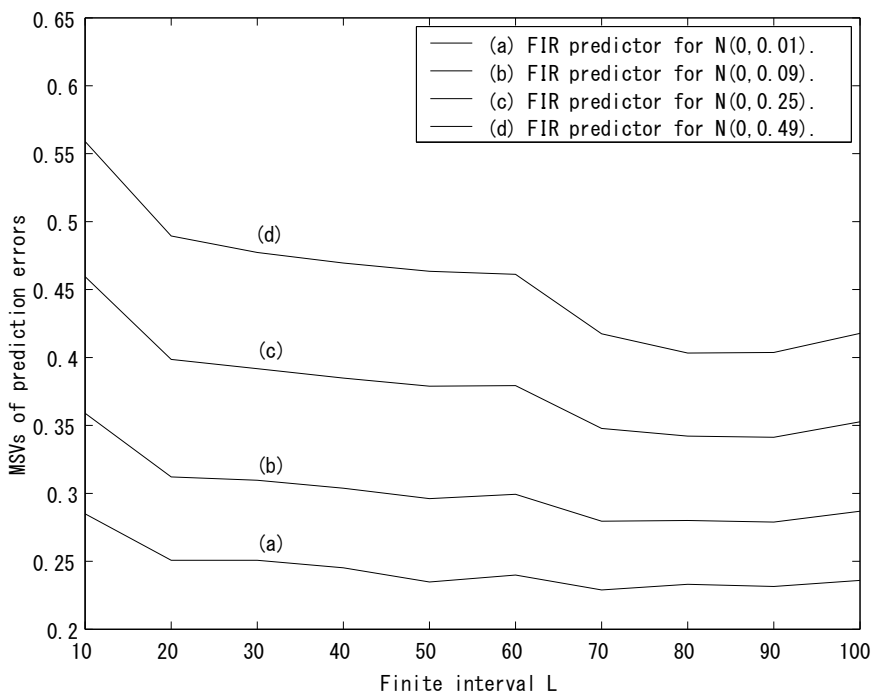

Figure 2. Mean-square values of the one-step ahead prediction errors $z(k+1)-\hat{z}(k+1 \mid k-L+1, k)$ by the RLS Wiener FIR predictor vs. the finite interval $L$ for the white Gaussian observation noises $N\left(0,0.1^{2}\right), N\left(0,0.3^{2}\right)$, $N\left(0,0.5^{2}\right)$ and $N\left(0,0.7^{2}\right)$. 


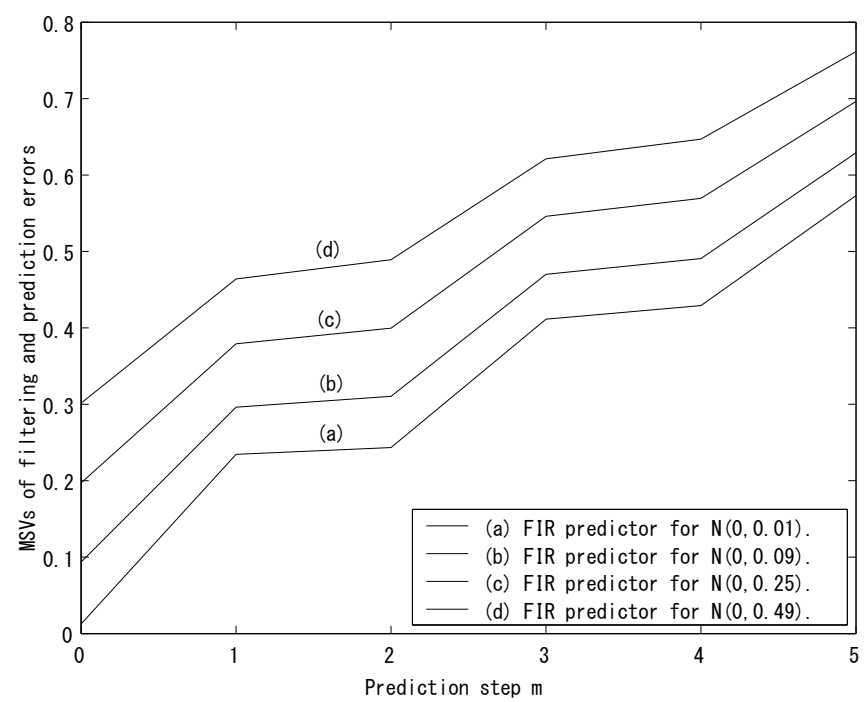

Figure 3. Mean-square values of the prediction errors $z(k+m)-\hat{z}(k+m \mid k-L+1, k), L=50$, by the proposed RLS Wiener FIR predictor vs. the prediction step $m, 1 \leq m \leq 5$, by the proposed RLS Wiener FIR predictor and the MSV of the filtering errors $z(k)-\hat{z}(k \mid k-L+1, k)$, in the case of $m=0$, by the proposed RLS Wiener FIR filter for the white Gaussian observation noises $N\left(0,0.1^{2}\right), N\left(0,0.3^{2}\right), N\left(0,0.5^{2}\right)$ and $N\left(0,0.7^{2}\right)$.

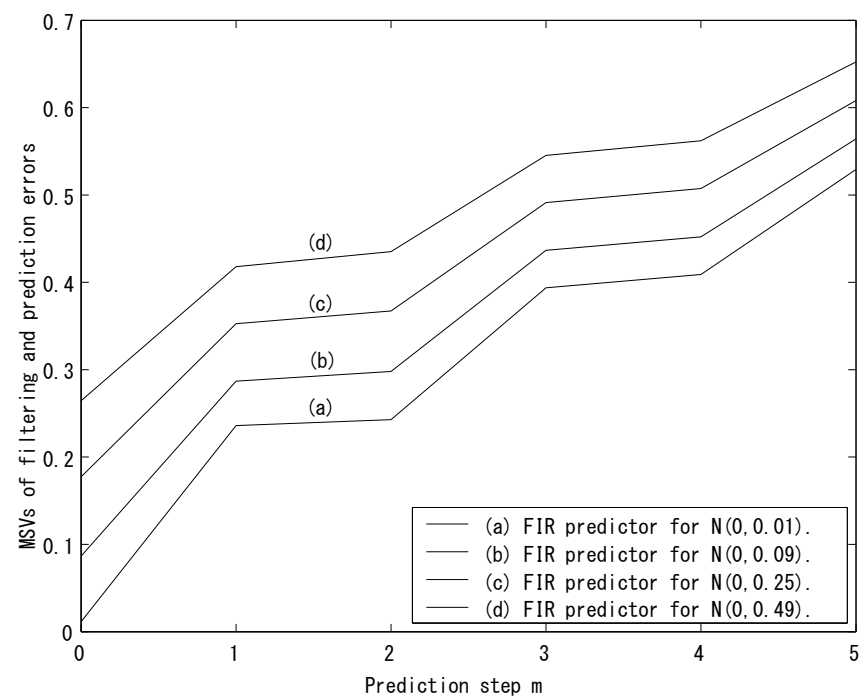

Figure 4. Mean-square values of the prediction errors $z(k+m)-\hat{z}(k+m \mid k-L+1, k), L=100$, vs. the prediction step $m, 1 \leq m \leq 5$, by the proposed RLS Wiener FIR predictor and the MSV of the filtering errors $z(k)-\hat{z}(k \mid k-L, k)$, in the case of $m=0$, by the proposed RLS Wiener FIR filter for the white Gaussian observation noises $N\left(0,0.1^{2}\right), N\left(0,0.3^{2}\right), N\left(0,0.5^{2}\right)$ and $N\left(0,0.7^{2}\right)$. 


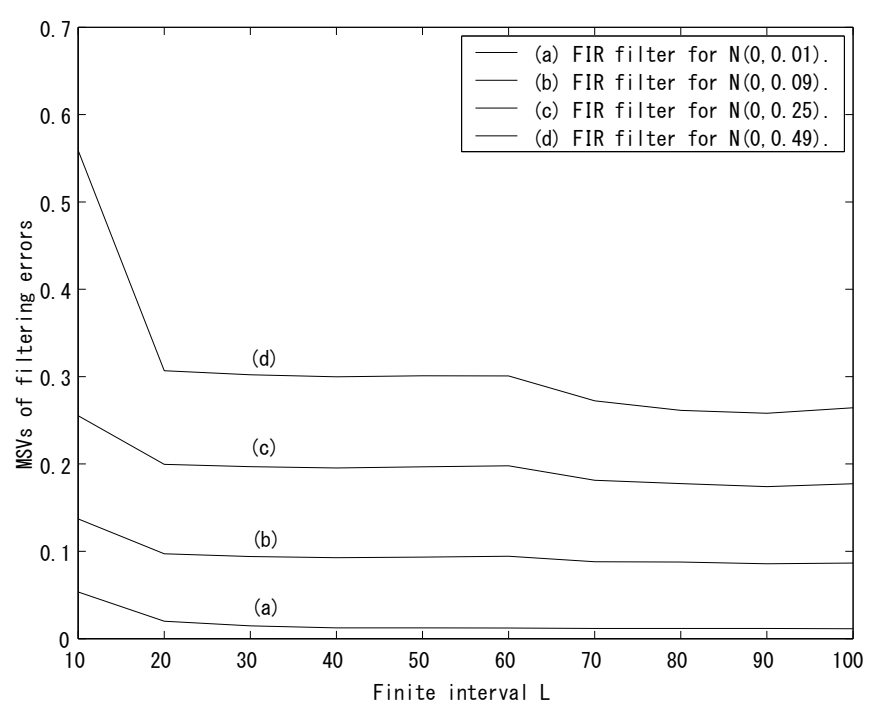

Figure 5. Mean-square values of the filtering errors $z(k)-\hat{z}(k \mid k-L+1, k)$ vs. the finite interval $L$ by the proposed RLS Wiener FIR filter for the white Gaussian observation noises $N\left(0,0.1^{2}\right), N\left(0,0.3^{2}\right), N\left(0,0.5^{2}\right)$ and $N\left(0,0.7^{2}\right)$.

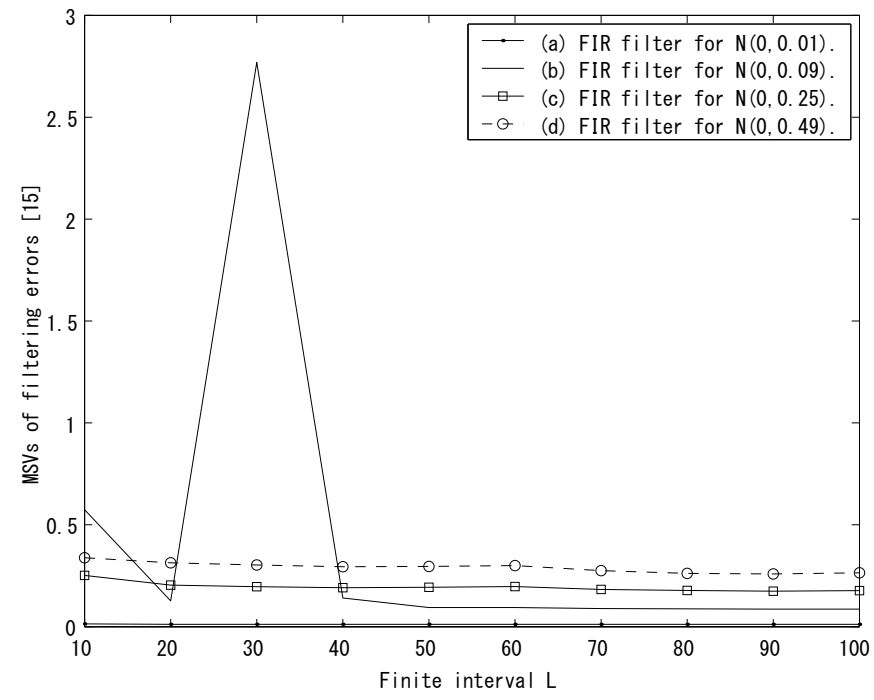

Figure 6. Mean-square values of the filtering errors $z(k)-\hat{z}(k \mid k-L, k)$ vs. the finite interval $L$ by the existing RLS Wiener FIR filter in [15] for the white Gaussian observation noises $N\left(0,0.1^{2}\right), N\left(0,0.3^{2}\right), N\left(0,0.5^{2}\right)$ and $N\left(0,0.7^{2}\right)$. 


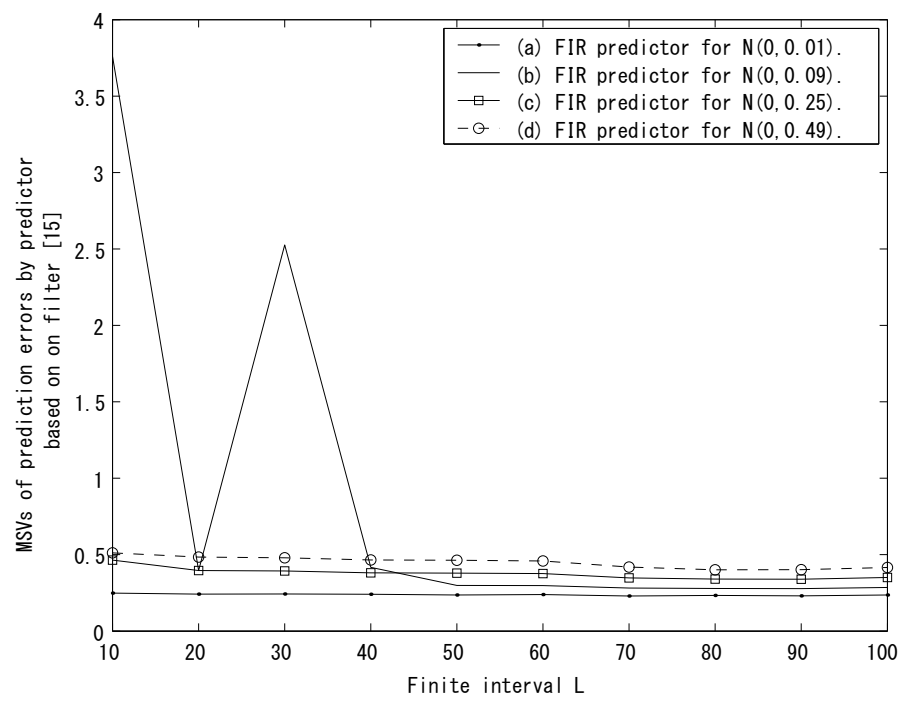

Figure 7. Mean-square values of the one-step ahead prediction errors $z(k+1)-\hat{z}(k+1 \mid k-L+1, k)$ by the RLS Wiener FIR predictor, obtained based on the RLS Wiener FIR filter in [15], vs. the finite interval $L$ for the white Gaussian observation noises $N\left(0,0.1^{2}\right), N\left(0,0.3^{2}\right), N\left(0,0.5^{2}\right)$ and $N\left(0,0.7^{2}\right)$.

the initial conditions, the proposed RLS Wiener FIR filter includes $N(N+1) / 2+N$ number of difference equations against $6 N^{2}+2 N$ in the RLS Wiener FIR filter in [15]. Hence, in the proposed RLS Wiener FIR filter and predictor, the computation times of the estimates are reduced in comparison with the RLS Wiener FIR filter in [15] and the RLS Wiener predictor, obtained from the RLS Wiener FIR filter in [15], respectively.

\section{References}

1. W. H. Kwon and O. K. Kwon, "FIR filters and recursive forms for continuous time-invariant state-space models," IEEE Trans. Automatic Control, vol. 32, no. 4, pp. 352-356, 1987.

2. A. H. Jazwinski, "Limited memory optimal filtering," IEEE Trans. Automatic Control, vol. 13, no. 5, pp. $558-563,1968$.

3. P. Maybeck, Stochastic Models, Estimation, and Control. New York: Academic, 1982.

4. F. C. Schweppe, Uncertain Dynamic Systems. New Jersey: Prentice-Hall, 1973.

5. A. M. Bruckstein and T. Kailath, "Recursive limited memory filtering and scattering theory," IEEE Trans. Information Theory, vol. 31, no. 3, pp. 440-443, 1985.

6. W. H. Kwon, P. S. Kim and P. G. Park, "A receding horizon kalman FIR filter for linear continuous-time systems," IEEE Trans. Automatic Control, vol. 44, no. 11, pp. 2115-2120, 1999.

7. S. H. Han, W. H. Kwon and P. S. Kim, "Receding-horizon unbiased FIR filter for continuous-time state-space models without a priori initial state informatio," IEEE Trans. Automatic Control, vol. 46, no. 5, pp. 766-770, 2001.

8. W. H. Kwon, P. S. Kim and P. G. Park, "A receding horizon kalman FIR filter for discrete time-invariant systems," IEEE Trans. Automatic Control, vol. 44, no. 9, pp. 1787-1791, 1999.

9. C. Ahn, "New quasi-deadbeat FIR smoother for discrete-time state-space signal models: an lmi approach," IEICE Trans. Fundam. Electron. Commun. Comput. Sci., vol. E91-A, no. 9, pp. 2671-2674, 2008.

10. C. K. Ahn and S. H. Han, "New $\mathrm{H}_{\infty}$ FIR smoother for linear discrete-time state-space models," IEICE Trans. Commun., vol. E91-B, no. 3, pp. 896-899, 2008.

11. S. Nakamori and M. Sugisaka, "Initial-value system for linear smoothing problems by covariance information," Automatica, vol. 13, no. 6, pp. 623-627, 1977.

12. S. Nakamori, "RLS fixed-lag smoother using covariance information in linear continuous stochastic systems," Appl. Math. Model, vol. 33, no. 1, pp. 242-255, 2009.

13. S. Nakamori, "Design of RLS Wiener FIR filter using covariance information in linear discrete-time stochastic systems," Digital Signal Processing, vol. 20, no. 5, pp. 1310-1329, 2010. 
14. S. Nakamori, "Design of RLS-FIR filter using covariance information in linear continuous-time stochastic system," Applied Mathematics and Computation, vol. 219, no. 17, pp. 9598-9608, 2013.

15. S. Nakamori, "Design of RLS Wiener FIR fixed-lag smoother in linear discrete-time stochastic systems," CiiT International Journal of Programmable Device Circuits and Systems, vol. 6, no. 8, pp. 233-243, 2014.

16. S. Nakamori, "Design of FIR smoother using covariance information for estimating signal at start time in linear continuous systems," Systems Science and Applied Mathematics, vol. 1, no. 3, pp. 29-37, 2016.

17. A. P. Sage and J. L. Melsa, Estimation Theory with Applications to Communications and Control. New York: McGraw-Hill, 1971.

18. S. Haykin, Adaptive Filter Theory (3rd Ed.). New Jersey: Prentice-Hall, 1996.

\section{Appendix: Proof of Theorem 1}

From the auto-covariance function (3) of the signal in the semi-degenerate kernel form, (9) is rewritten as

$$
g(k, s) \Lambda(s)=\alpha(k+m) \beta^{T}(s) H^{T}-\sum_{i=s-1-L+1}^{s-1} g(k, i) \Lambda(i) g_{f}^{T}(s-1, i) \Phi^{T} H^{T}
$$

Let us introduce the function $J(s)$, which satisfies

$$
J(s) \Lambda(s)=\beta^{T}(s) H^{T}-\sum_{i=s-1-L+1}^{s-1} J(i) \Lambda(i) g_{f}^{T}(s-1, i) \Phi^{T} H^{T}
$$

From (A-1) and (A-2), the impulse response function $g(k, s)$ is given by

$$
g(k, s)=\alpha(k+m) J(s) .
$$

Similarly, from (10), the impulse response function $g_{f}(k, s)$ is given by

$$
g_{f}(k, s)=\alpha(k) J(s) .
$$

By introducing a function

$$
r(k)=\sum_{i=k-L+1}^{k} J(i) \Lambda(i) J^{T}(i),
$$

$J(k)$ satisfies

$$
J(k)=\left(\beta^{T}(k) H^{T}-r(k-1) \alpha^{T}(k-1) \Phi^{T} H^{T}\right) \Lambda^{-1}(k) .
$$

From (A-5), the difference equation for $r(k)$ is written as

$$
r(k)=r(k-1)+J(k) \Lambda(k) J^{T}(k)-J(k-L) \Lambda(k-L) J^{T}(k-L) .
$$

From (5) and (A-4), the filtering estimate is given by

$$
\hat{x}_{f}(k \mid k-L+1, k)=\alpha(k) e(k) .
$$

Here, the function $e(k)$ satisfies

$$
e(k)=\sum_{i=k-L+1}^{k} J(i) \nu(i)
$$

From (A-9), the difference equation for $e(k)$ is given by

$$
\begin{aligned}
& e(k)=e(k-1)+J(k)\left(y(k)-H \Phi \hat{x}_{f}(k-1 \mid k-1-L+1, k-1)\right) \\
& -J(k-L)\left(y(k-L)-H \Phi \hat{x}_{f}(k-L-1 \mid k-L-1-L+1, k-L-1)\right) .
\end{aligned}
$$


From the auto-variance function of the innovation process $\nu(s), \Lambda(s)=E\left[\nu(s) \nu^{T}(s)\right], \Lambda(k)$ is expanded as follows.

$$
\begin{aligned}
& \Lambda(k)=E\left[\nu(k) \nu^{T}(k)\right] \\
& =E\left[\left(y(k)-H \Phi \hat{x}_{f}(k-1 \mid k-1-L+1, k-1)\right)\left(y(k)-H \Phi \hat{x}_{f}(k-1 \mid k-1-L+1, k-1)\right)^{T}\right] \\
& =K(k, k)+R-H \Phi E\left[\hat{x}_{f}(k-1 \mid k-1-L+1, k-1) \hat{x}_{f}^{T}(k-1 \mid k-1-L+1, k-1)\right] \Phi^{T} H^{T} \\
& =K(k, k)+R-H \Phi S(k-1) \Phi^{T} H^{T}
\end{aligned}
$$

Here, we put the auto-variance function $S(k)$ of the filtering estimate $\hat{x}_{f}(k \mid k-L+1, k)$ as $S(k)=$ $E\left[\hat{x}_{f}(k \mid k-L+1, k) \hat{x}_{f}^{T}(k \mid k-L+1, k)\right]$. From (5), (A-4) and (A-5), the function $S(k)$ is expanded as follows.

$$
\begin{aligned}
& S(k)=E\left[\hat{x}_{f}(k \mid k-L+1, k) \hat{x}_{f}^{T}(k \mid k-L+1, k)\right] \\
& =\sum_{i=k-L+1}^{k} g_{f}(k, i) E\left[\nu(i) \nu^{T}(i)\right] g_{f}^{T}(k, i) \\
& =\sum_{i=k-L+1}^{k} g_{f}(k, i) \Lambda(i) g_{f}^{T}(k, i) \\
& =\alpha(k) \sum_{i=k-L+1}^{k} J(i) \Lambda(i) J^{T}(i) \alpha^{T}(k) \\
& =\alpha(k) r(k) \alpha^{T}(k)
\end{aligned}
$$

By substituting (A-7) into (A-12), from (3) and (A-4), the difference equation for $S(k)$ is developed as follows.

$$
\begin{aligned}
& S(k)=\alpha(k)\left(r(k-1)+J(k) \Lambda(k) J^{T}(k)-J(k-L) \Lambda(k-L) J^{T}(k-L)\right) \alpha^{T}(k) \\
& =\Phi S(k-1) \Phi^{T}+g_{f}(k, k) \Lambda(k) g_{f}^{T}(k, k) \\
& -\Phi^{L} g_{f}(k-L, k-L) \Lambda(k-L) g_{f}^{T}(k-L, k-L)\left(\Phi^{T}\right)^{L}
\end{aligned}
$$

From (A-8) and (A-10), the difference equation for the filtering estimate is derived as follows.

$$
\begin{aligned}
& \hat{x}_{f}(k \mid k-L+1, k)=\Phi \hat{x}_{f}(k-1 \mid k-L, k-1) \\
& +g_{f}(k, k)\left(y(k)-H \Phi \hat{x}_{f}(k-1 \mid k-1-L+1, k-1)\right) \\
& -\Phi^{L} g_{f}(k-L, k-L)\left(y(k-L)-H \Phi \hat{x}_{f}(k-L-1 \mid k-L-1-L+1, k-L-1)\right)
\end{aligned}
$$

From (A-4) and (A-6), the filter gain $g_{f}(k, k)$ is obtained as

$$
g_{f}(k, k)=\left(K(k, k) H^{T}-\Phi S(k-1) \Phi^{T} H^{T}\right) \Lambda^{-1}(k) .
$$

From (4), (5), (A-3) and (A-4), the m-step ahead prediction estimate $\hat{x}(k+m \mid k-L+1, k)$ is related to the filtering estimate $\hat{x}_{f}(k \mid k-L+1, k)$ as

$$
\hat{x}(k+m \mid k-L+1, k)=\Phi^{m} \hat{x}_{f}(k \mid k-L+1, k) .
$$

Now, from (5), the initial condition on the difference equation (14) for the FIR filtering estimate $\hat{x}_{f}(k \mid k-L+1, k)$ at $k=L, \hat{x}_{f}(L \mid 1, L)$, is expressed as

$$
\begin{aligned}
& \hat{x}_{f}(L \mid 1, L)=\sum_{i=1}^{L} g_{f}(L, i) \bar{\nu}(i), \\
& \bar{\nu}(i)=y(L)-H \Phi \hat{\bar{x}}_{f}(L-1 \mid 1, L-1) .
\end{aligned}
$$

We denote the initial conditions $\hat{x}_{f}(L, 1, L)$ as $\hat{\bar{x}}_{f}(L, 1, L)$ and $g_{f}(L, s)$ as $\bar{g}_{f}(L, s)$ respectively. From $(\mathrm{A}-4), \bar{g}_{f}(L, s)$ satisfies

$$
\bar{g}_{f}(L, s)=\alpha(L) \bar{J}(s) .
$$

Here, from $(\mathrm{A}-2), \bar{J}(s)$ satisfies

$$
\bar{J}(s) \bar{\Lambda}(s)=\beta^{T}(s) H^{T}-\sum_{i=1}^{s-1} \bar{J}(i) \bar{\Lambda}(i) \bar{g}_{f}^{T}(s-1, i) \Phi^{T} H^{T} .
$$


From (A-17) and (A-18), the filtering estimate $\hat{\bar{x}}_{f}(L \mid 1, L)$ is given by

$$
\begin{aligned}
& \hat{\bar{x}}_{f}(L \mid 1, L)=\alpha(L) \sum_{i=1}^{L} \bar{J}(i) \bar{\nu}(i) \\
& =\alpha(L) \bar{e}(L) .
\end{aligned}
$$

Here, the function $\bar{e}(L)$ is introduced.

$$
\bar{e}(L)=\sum_{i=1}^{L} \bar{J}(i) \bar{\nu}(i)
$$

From (A-21), it follows that

$$
\bar{e}(L)=\bar{e}(L-1)+\bar{J}(L)\left(y(L)-H \Phi \hat{\bar{x}}_{f}(L-1 \mid 1, L-1)\right) .
$$

Substituting (A-22) into (A-20) and using (A-18), we have

$$
\begin{aligned}
& \hat{\bar{x}}_{f}(L \mid 1, L)=\alpha(L) \bar{e}(L) \\
& =\Phi \hat{\bar{x}}_{f}(L-1 \mid 1, L-1)+\alpha(L) \bar{J}(L)\left(y(L)-H \Phi \hat{\bar{x}}_{f}(L-1 \mid 1, L-1)\right) \\
& =\Phi \hat{\bar{x}}_{f}(L-1 \mid 1, L-1)+\bar{g}_{f}(L, L)\left(y(L)-H \Phi \hat{\bar{x}}_{f}(L-1 \mid 1, L-1)\right), \\
& \hat{\bar{x}}_{f}(0 \mid 1,0)=0 .
\end{aligned}
$$

From (A-18) and (A-19), the filter gain $\mathrm{g} \bar{g}_{f}(L, L)$ is formulated as

$$
\begin{aligned}
& \bar{g}_{f}(L, L)=\left(K(L, L) H^{T}-\alpha(L) \sum_{i=1}^{L-1} \bar{J}(i) \bar{\Lambda}(i) \bar{g}_{f}^{T}(L-1, i) \Phi^{T} H^{T}\right) \bar{\Lambda}^{-1}(L) \\
& =\left(K(L, L) H^{T}-\Phi^{L} \sum_{i=1}^{L-1} \bar{J}(i) \bar{\Lambda}(i) \bar{J}^{T}(i)\left(\Phi^{T}\right)^{L} H^{T}\right) \bar{\Lambda}^{-1}(L) \\
& =\left(K(L, L) H^{T}-\Phi^{L} \bar{r}(L-1)\left(\Phi^{T}\right)^{L} H^{T}\right) \bar{\Lambda}^{-1}(L) \\
& =\left(K(L, L) H^{T}-\Phi \bar{S}(L-1) \Phi^{T} H^{T}\right) \bar{\Lambda}^{-1}(L)
\end{aligned}
$$

Here, we introduced the function $\bar{r}(L)$ and $\bar{S}(L)$, which are given by

$$
\begin{aligned}
\bar{r}(L) & =\sum_{i=1}^{L} \bar{J}(i) \bar{\Lambda}(i) \bar{J}^{T}(i), \\
\bar{S}(L) & =\Phi^{L} \bar{r}(L)\left(\Phi^{T}\right)^{L} .
\end{aligned}
$$

The difference equation for $\bar{r}(L)$ is developed as

$$
\bar{r}(L)=\bar{r}(L-1)+\bar{J}(L) \bar{\Lambda}(L) \bar{J}^{T}(L), \bar{r}(0)=0 .
$$

Substituting (A-26) into $\bar{S}(L)=\Phi^{L} \bar{r}(L)\left(\Phi^{T}\right)^{L}$, we have the difference equation for $\bar{S}(L)$ as follows.

$$
\bar{S}(L)=\Phi \bar{S}(L-1) \Phi^{T}+\bar{g}_{f}(L, L) \bar{\Lambda}(L) \bar{g}_{f}^{T}(L, L), \bar{S}(0)=0
$$

Finally, from the auto-variance function of the innovation process $\bar{\nu}(L), \bar{\Lambda}(L)=E\left[\bar{\nu}(L) \bar{\nu}^{T}(L)\right], \bar{\Lambda}(L)$ is expanded as follows.

$$
\begin{aligned}
& \bar{\Lambda}(L)=E\left[\bar{\nu}(L) \bar{\nu}^{T}(L)\right] \\
& =E\left[\left(\bar{y}(L)-H \Phi \hat{\bar{x}}_{f}(L-1 \mid 1, L-1)\right)\left(y(k)-H \Phi \hat{\bar{x}}_{f}(L-1 \mid 1, L-1)\right)^{T}\right] \\
& =H K(L, L) H^{T}+R-H \Phi E\left[\hat{\bar{x}}_{f}(L-1 \mid 1, L-1) \hat{\bar{x}}_{f}^{T}(L-1 \mid 1, L-1)\right] \Phi^{T} H^{T} \\
& =H K(L, L) H^{T}+R-H \Phi \bar{S}(L-1) \Phi^{T} H^{T}
\end{aligned}
$$

(Q.E.D.) 